\title{
CUSP TRANSITIVITY IN HYPERBOLIC 3-MANIFOLDS
}

\author{
JOHN G. RATCLIFFE AND STEVEN T. TSCHANTZ
}

\begin{abstract}
In this paper, we study multiply transitive actions of the group of isometries of a cusped finite-volume hyperbolic 3-manifold on the set of its cusps. In particular, we prove a conjecture of Vogeler that there is a largest $k$ for which such $k$-transitive actions exist, and that for each $k \geq 3$, there is an upper bound on the possible number of cusps.
\end{abstract}

\section{INTRODUCTION}

Let $S$ be a set and $k$ an integer such that $1 \leq k \leq|S|$. An action of a group $G$ on $S$ is called $k$-transitive if for every choice of distinct elements $x_{1}, \ldots, x_{k}$ of $S$ and every choice of distinct targets $y_{1}, \ldots, y_{k}$ in $S$, there is an element $g$ of $G$ such that $g x_{i}=y_{i}$ for each $i=1, \ldots, k$. The term transitive means 1-transitive, and actions with $k>1$ are called multiply transitive.

This paper is concerned with cusped hyperbolic 3-manifolds of finite volume whose group of isometries induces a multiply transitive action on the set of cusps of the manifold. Call such a manifold $k$-transitive if the induced action is $k$-transitive. All 3-manifolds in this paper are connected.

Multiply transitive cusped hyperbolic 3-manifolds of finite volume have a high degree of symmetry and are therefore are some of the most beautiful cusped hyperbolic 3-manifolds of finite volume.

Examples of $k$-transitive hyperbolic link complements are described by Vogeler in [13] for $k=1,2,3,4$; moreover, Vogeler proved that there is no upper bound for the number of cusps for a 2-transitive hyperbolic 3-manifold of finite volume. Vogeler conjectured that there is a largest $k$ for which $k$-transitive manifolds exist, and for each $k \geq 3$ there is an upper bound on the possible number of cusps. In this paper, we prove Vogeler's conjecture; more precisely, we prove the following theorem:

Theorem 1. Let $M$ be a $k$-transitive cusped hyperbolic 3-manifold of finite volume. Then the possible values of $k$ are 1,2,3,4 and 5; moreover,

(1) if $M$ is 5 -transitive, then $M$ has exactly 5 cusps,

(2) if $M$ is 4-transitive and not 5-transitive, then $M$ has exactly 4 cusps,

(3) if $M$ is 3-transitive and not 4-transitive, then the possible numbers of cusps of $M$ are $3,5,6$ and 8.

\section{Preliminary lemmas}

Let $C$ be a cusp of a hyperbolic 3-manifold $M$ of finite volume. A link $L$ of $C$ is a horospherical cross-section of $C$. A link $L$ of $C$ has a natural Euclidean metric, and so $L$ is either a flat torus or a flat Klein bottle. We say that $C$ is orientable if $L$ is a torus and $C$ is nonorientable if $L$ is a Klein bottle. If $M$ is orientable, then each cusp of $M$ is orientable. 
Lemma 1. Let $G$ be the group of isometries of a cusped hyperbolic 3-manifold $M$ of finite volume that stabilizes a cusp $C$ of $M$. Then $G$ acts effectively on each link $L$ of $C$ as a group of isometries with respect to the natural Euclidean metric on $L$.

Proof. We shall work in the upper half-space model $U^{3}$ of hyperbolic 3 -space. Then there is a discrete subgroup $\Gamma$ of the group $\mathrm{M}\left(U^{3}\right)$ of Möbius transformations of $\hat{E}^{3}$ that leave $U^{3}$ invariant such that $M=U^{3} / \Gamma$. Let $L$ be a link of the cusp $C$. Then there is a point $c$ on the sphere $\hat{E}^{2}$ at infinity that is fixed by a parabolic element $f$ of $\Gamma$, and there is a maximal open horoball $B$ based at $c$ such that $B$ is precisely invariant by the stabilizer $\Gamma_{c}$ in $\Gamma$ of the point $c$, and the inclusion of $B$ into $U^{3}$ induces a local isometry of $B / \Gamma_{c}$ onto $C$; moreover, there is a horosphere $\Sigma$ based at $c$ and contained in $B$ such that the inclusion of $B$ into $U^{3}$ induces an isometry from $\Sigma / \Gamma_{c}$ to $L$ where $\Sigma$ has the natural Euclidean metric (see p. 139 of [9]) and $L$ has the induced Euclidean metric. The point $c$ and the horoball $B$ are unique up to the action of $\Gamma$ on $\hat{E}^{2}$.

Let $g$ be an isometry of $M$ that stabilizes the cusp $C$. Then $g$ lifts to an element $\tilde{g}$ of $\mathrm{M}\left(U^{3}\right)$ such that $\tilde{g} \Gamma \tilde{g}^{-1}=\Gamma$ by Theorem 13.2.6 of [9]. In particular $\tilde{g} f \tilde{g}^{-1}$ is a parabolic element of $\Gamma$ that fixes the point $\tilde{g} c$. As $g$ stabilizes the cusp $C$, we have that $\tilde{g} c=h c$ for some element $h$ of $\Gamma$. By replacing $\tilde{g}$ by $h^{-1} \tilde{g}$, we may assume that $\tilde{g} c=c$. Now $\tilde{g}$ is either an elliptic or parabolic transformation by Theorem 5.5.4 of [9]. Therefore $\tilde{g}$ leaves $\Sigma$ invariant and $\tilde{g}$ acts on $\Sigma$ as an isometry by Theorem 4.7.4 of [9]. Hence $g$ acts on $L$ as an isometry with respect to the natural Euclidean metric on $L$.

By conjugating $\Gamma$ in $\mathrm{M}\left(U^{3}\right)$, we may assume that $c=\infty$. Then the action of $\tilde{g}$ on $E^{2}$, as a Euclidean isometry, determines the action of $\tilde{g}$ on $U^{3}$ in a parallel fashion on each horizontal horosphere by Poincaré extension (see $\S 4.4$ of [9]). Hence, if $g$ acts trivially on $L$, then $g$ acts trivially on the open subset $C$ of $M$, and therefore $g$ is trivial. Thus $G$ acts effectively on $L$.

Lemma 2. Let

$$
A=\left(\begin{array}{rr}
-1 & 0 \\
0 & 1
\end{array}\right), \quad B=\left(\begin{array}{rr}
-1 & 1 \\
0 & 1
\end{array}\right), \quad C=\left(\begin{array}{ll}
0 & 1 \\
1 & 0
\end{array}\right) .
$$

The group $\mathrm{GL}(2, \mathbb{Z})$ is the free product of the dihedral group $\langle A, C\rangle$ of order 8 and the dihedral group $\langle B, C\rangle$ of order 12 amalgamated along the dihedral subgroup $\langle-I, C\rangle$ of order 4 . Every finite subgroup of $\mathrm{GL}(2, \mathbb{Z})$ is conjugate to a subgroup of $\langle A, C\rangle$ or $\langle B, C\rangle$.

Proof. The matrices $A, B, C$ have order 2 , and $(A C)^{2}=-I$, and $(B C)^{3}=-I$. Hence $\langle A, C\rangle$ is a dihedral group of order 8 , and $\langle B, C\rangle$ is a dihedral group of order 12 .

The group $\operatorname{PGL}(2, \mathbb{Z})$ acts effectively by isometries on the upper complex halfspace model of hyperbolic 2 -space $H^{2}$ by

$$
\pm\left(\begin{array}{cc}
a & b \\
c & c
\end{array}\right) z=\frac{a z+b}{c z+d} \text { if } \operatorname{det}\left(\begin{array}{cc}
a & b \\
c & c
\end{array}\right)=1,
$$

and

$$
\pm\left(\begin{array}{cc}
a & b \\
c & c
\end{array}\right) z=\frac{a \bar{z}+b}{c \bar{z}+d} \text { if } \operatorname{det}\left(\begin{array}{cc}
a & b \\
c & c
\end{array}\right)=-1,
$$

It is well know that a fundamental domain for the action of $\operatorname{PGL}(2, \mathbb{Z})$ on $H^{2}$ is the hyperbolic triangle $T$ with vertices $i, \frac{1}{2}+\frac{\sqrt{3}}{2} i, \infty$ and corresponding angles 
$\pi / 2, \pi / 3,0$. Moreover $\mathrm{PGL}(2, \mathbb{Z})$ is a triangle reflection group with respect to the reflections $R_{1}(z)=-\bar{z}, R_{2}(z)=1 / \bar{z}, R_{2}(z)=1-\bar{z}$ in the sides of $T$. Note that $R_{1}$ is the reflection in the $y$-axis, $R_{2}$ is the inversion in the circle $|z|=1$, and $R_{3}$ is the reflection in the line $x=\frac{1}{2}$. Moreover

$$
( \pm A) z=R_{1}(z), \quad( \pm C) z=R_{2}(z), \quad( \pm B) z=R_{3}(z) .
$$

By Poincaré's fundamental polygon theorem (Theorem 13.5.3 [9]), the group $\operatorname{PGL}(2, \mathbb{Z})$ has the presentation

$$
\left\langle R_{1}, R_{2}, R_{3} ; R_{1}^{2}, R_{2}^{2}, R_{3}^{2},\left(R_{1} R_{2}\right)^{2},\left(R_{2} R_{3}\right)^{3}\right\rangle,
$$

which is equivalent to the presentation

$$
\left\langle R_{1}, R_{2}, R_{3}, R_{4} ; R_{1}^{2}, R_{2}^{2},\left(R_{1} R_{2}\right)^{2}, R_{2}=R_{4}, R_{3}^{2}, R_{4}^{2},\left(R_{4} R_{3}\right)^{3}\right\rangle .
$$

Therefore $\operatorname{PGL}(2, \mathbb{Z})$ is the free product with amalgamation of the dihedral group $\left\langle R_{1}, R_{2}\right\rangle$ of order 4 and the dihedral group $\left\langle R_{2}, R_{3}\right\rangle$ of order 6 amalgamated along the subgroup $\left\langle R_{2}\right\rangle$ of order 2 .

Let $\eta: \mathrm{GL}(2, \mathbb{Z}) \rightarrow \operatorname{PGL}(2, \mathbb{Z})$ the quotient map. Then $\mathrm{GL}(2, \mathbb{Z})$ acts on the Bass-Serre tree of the amalgamated product decomposition of $\operatorname{PGL}(2, \mathbb{Z})$ via $\eta$. Hence $\mathrm{GL}(2, \mathbb{Z})$ is the amalgamated product of the groups

$$
\eta^{-1}\left(\left\langle R_{1}, R_{2}\right\rangle\right)=\langle A, C\rangle \text { and } \eta^{-1}\left(\left\langle R_{2}, R_{3}\right\rangle\right)=\langle B, C\rangle
$$

along the subgroup $\eta^{-1}\left(\left\langle R_{2}\right\rangle\right)=\langle-I, C\rangle$. By the torsion theorem for amalgamated products of groups, every finite subgroup of $\mathrm{GL}(2, \mathbb{Z})$ is conjugate to a subgroup of $\langle A, C\rangle$ or $\langle B, C\rangle$

\section{On $k$-Transitive Manifolds with $k \geq 5$}

Our basic reference for the theory of finite permutation groups is Wielandt's classic book [14]. In particular, we refer to [14] for all basic definitions in the elementary theory of permutation groups.

Lemma 3. If $G$ is a 4-transitive permutation group of degree $n$ with a nontrivial solvable normal subgroup, then $n=4$ and $G=S_{4}$.

Proof. The group $G$ is primitive by Theorem 9.6 of [14]. Let $N$ be a minimal, nontrivial, solvable, normal subgroup of $G$. Then $N$ is regular by Theorem 11.5 of [14]. Hence $n=4$ by Theorem 11.3(d) of [14]. Therefore $G=S_{4}$.

Theorem 2. Let $M$ be a cusped hyperbolic 3-manifold $M$ of finite volume such that the induced action of the group of isometries of $M$ on the set of cusps of $M$ is $k$-transitive with $k \geq 5$. Then $k=5$, and the possible number of cusps of $M$ is 5; moreover, each cusp of $M$ is orientable.

Proof. Let $G=\operatorname{Isom}(M)$. It is well known that $G$ is finite (see Theorem 12.7.7 of [9]). Let $\Omega$ be the set of cusps of $M$, and let $G^{\Omega}$ be the induced permutation group of $\Omega$. Let $n=|\Omega|$. As $5 \leq k \leq n$, we have that $n \geq 5$. As $G^{\Omega}$ acts transitively on $\Omega$, the degree of $G^{\Omega}$ is $n$. Fix a cusp $C$ of $M$, and let $G_{1}$ be the subgroup of $G$ that stabilizes $C$, and let $G_{1}^{\Omega}$ be the corresponding point stabilizer of $G^{\Omega}$.

Let $L$ be a link of $C$. By Lemma 1, we may regard $G_{1}$ to be a subgroup of the group $\operatorname{Aff}(L)$ of affine transformations of $L$. Assume first that $L$ is a torus. By Theorems 1 and 3 of [10], there is a short exact sequence

$$
1 \rightarrow \mathbb{R}^{2} / \mathbb{Z}^{2} \rightarrow \operatorname{Aff}(L) \stackrel{\pi}{\longrightarrow} \mathrm{GL}(2, \mathbb{Z}) \rightarrow 1 .
$$


Then $\pi\left(G_{1}\right)$ is a finite subgroup of $\mathrm{GL}(2, \mathbb{Z})$. Therefore $\pi\left(G_{1}\right)$ is solvable by Lemma 2. Hence $G_{1}$ is solvable, since $\operatorname{ker}(\pi)$ is abelian.

The group $G_{1}$ maps onto $G_{1}^{\Omega}$, and so $G_{1}^{\Omega}$ is solvable. Now $G_{1}^{\Omega}$ is $(k-1)$-transitive on $\Omega-\{C\}$ by Theorem 9.1 of [14]. Hence, the degree of $G_{1}^{\Omega}$ is $n-1$. As $G_{1}^{\Omega}$ is 4 transitive, $G_{1}^{\Omega}$ has degree 4 and $G_{1}^{\Omega}=S_{4}$ by Lemma 3 . Hence $n-1=4$, and so $n=5$. Therefore $M$ has five cusps. Moreover $k=5$, since $5 \leq k \leq n$. For an example of such a manifold, see Example 1 in $\S 6$.

Now assume that $L$ is a Klein bottle. By Theorems 1 and 3 of [10] and Exercise 9.5.8 of [9], there is a short exact sequence

$$
1 \rightarrow \mathbb{R} / \mathbb{Z} \rightarrow \operatorname{Aff}(L) \stackrel{\pi}{\longrightarrow}(\mathbb{Z} / 2 \mathbb{Z})^{2} \rightarrow 1 .
$$

Clearly, $G_{1}$ is solvable. The same argument as in the case that $L$ is a torus implies that $G_{1}^{\Omega}=S_{4}$.

Let $K$ be the kernel of the projection from $\operatorname{Aff}(L)$ to $(\mathbb{Z} / 2 \mathbb{Z})^{2}$. Then $K \cap G_{1}$ is an abelian normal subgroup of $G_{1}$ that projects onto a normal subgroup $N$ of $G_{1}^{\Omega}$ of order 1 or 4 , since $S_{4}$ has a unique abelian proper normal subgroup, and this subgroup has order 4 . Therefore $G_{1} /\left(K \cap G_{1}\right) \cong\left(G_{1} K\right) / K$ has order at most 4 and projects onto the group $G_{1}^{\Omega} / N$ of order at least 6 , which is a contradiction. Thus each cusp of $M$ is orientable.

\section{On 4-Transitive Manifolds that are not 5-Transitive}

Lemma 4. Let $G$ be a 2-transitive permutation group of degree $n$ with a nontrivial solvable normal subgroup. Then

(1) The group $G$ has a nontrivial, regular, abelian, normal subgroup $N$.

(2) The group $N$ is an elementary abelian p-group for some prime number $p$.

(3) The order of $N$ is $n$, and so $n=p^{m}$ for some positive integer $m$.

(4) The group $N$ is the unique minimal nontrivial normal subgroup of $G$.

(5) The group $N$ is the unique nontrivial abelian normal subgroup of $G$.

Proof. The group $G$ is primitive by Theorem 9.6 of [14]. Let $N$ be a minimal nontrivial solvable normal subgroup of $G$. Then $N$ is regular, $N$ is an elementary abelian group, and the only nontrivial minimal normal subgroup of $G$ by Theorem 11.5 of [14]. Hence $n=|N|$ by Proposition 4.2 of [14].

Suppose $A$ is a nontrivial abelian normal subgroup of $G$. The group $A$ is transitive by Theorem 8.8 of [14]. The group $A$ is regular by Proposition 4.4 of [14], and so $n=|A|$ by Proposition 4.2 of [14]. As the minimal nontrivial normal subgroup of $G$ contained in $A$ is $N$, we have that $A=N$.

Lemma 5. Let $G$ be a 3-transitive permutation group of degree $n$ with a nontrivial solvable normal subgroup. Then either $n=3$ and $G=S_{3}$ or $n=2^{m}$ for some integer $m \geq 2$.

Proof. This follows from Lemma 4(1), Theorem 11.3(b) of [14], and $3 \leq n$.

Lemma 6. Let $H$ be a finite subgroup of $\mathbb{R}^{n} / \mathbb{Z}^{n}$ for some positive integer $n$. Then the rank (minimal number of generators) of $H$ is at most $n$.

Proof. Let $\eta: \mathbb{R}^{n} \rightarrow \mathbb{R}^{n} / \mathbb{Z}^{n}$ be the quotient map. Then $\mathbb{Z}^{n}$ is a finite index subgroup of $\eta^{-1}(H)$. Hence $\eta^{-1}(H)$ is a discrete subgroup of $\mathbb{R}^{n}$. Therefore $\eta^{-1}(H)$ is generated by a set of linearly independent vectors $\left\{v_{1}, \ldots, v_{m}\right\}$ with $m \leq n$ by Theorem 5.3.2 of [9]. Therefore $H$ is generated by $\left\{\eta\left(v_{1}\right), \ldots, \eta\left(v_{m}\right)\right\}$. 
Theorem 3. Let $M$ be a cusped hyperbolic 3-manifold of finite volume such that the induced action of the group of isometries of $M$ on the set of its cusps is 4-transitive but not 5-transitive. Then the possible number of cusps of $M$ is 4 .

Proof. We continue with the notation in the proof of Theorem 2. Then as in the proof of Theorem 1, we have that $G_{1}^{\Omega}$ is solvable and $G_{1}^{\Omega}$ is 3-transitive on $\Omega-\{C\}$. By Lemma 5 , the degree of $G_{1}^{\Omega}$ is 3 or $2^{m}$ for some integer $m \geq 2$. In the first case, the degree of $G^{\Omega}$ is 4 by Theorem 9.1 of [14]. Therefore $M$ has exactly 4 cusps.

Now assume that the second case holds. Then the degree of $G_{1}^{\Omega}$ is at least 4 . Hence, the order of $G_{1}^{\Omega}$ is at least 24 by Theorem 9.3 of [14]. Let $K$ be the kernel of the projection $\pi: \operatorname{Aff}(L) \rightarrow\left(\operatorname{GL}(2, \mathbb{Z})\right.$ or $\left.(\mathbb{Z} / 2 \mathbb{Z})^{2}\right)$. Then $K \cap G_{1}$ is an abelian normal subgroup of $G_{1}$ that projects to an abelian normal subgroup $N$ of $G_{1}^{\Omega}$. Assume first that $N$ is trivial. Then the finite subgroup $G_{1} /\left(K \cap G_{1}\right) \cong G_{1} K / K$ of $\operatorname{GL}(2, \mathbb{Z})$ or $(\mathbb{Z} / 2 \mathbb{Z})^{2}$ projects onto $G_{1}^{\Omega}$. Hence, the order of $G_{1}^{\Omega}$ is at most 12 by Lemma 2, which is a contradiction. Therefore $N$ is nontrivial.

The group $N$ is an elementary 2-group of rank at least 2 by Lemmas 4 and 5 . The group $K \cap G_{1}$ has rank at most 2 by Lemma 6 . Hence, the rank of $N$ is at most 2. Therefore, the rank of $N$ is 2 and the degree of $G_{1}^{\Omega}$ is 4 . Hence, $G_{1}^{\Omega}=S_{4}$, since $G_{1}^{\Omega}$ has order at least 24 . Therefore $G^{\Omega}$ is 5 -transitive by Theorem 9.1 of [14]. However, $G^{\Omega}$ is not 5 -transitive by assumption, and so the second case does not occur. Therefore $M$ has exactly 4 cusps. For an example of such a manifold, see Example 2 in $\S 6$.

\section{On 3-Transitive Manifolds that are nOt 4-Transitive}

Theorem 4. Let $M$ be a cusped hyperbolic 3-manifold of finite volume such that the induced action of the group of isometries of $M$ on the set of its cusps is 3-transitive but not 4-transitive. Then the possible numbers of cusps of $M$ are 3,5,6 and 8, and the corresponding permutation groups are $S_{3}, A_{5}, \mathrm{PGL}(2,5)$ and $\mathrm{PGL}(2,7)$ respectively, of orders $6,60,120$ and 336 respectively.

Proof. We continue with the notation in the proofs of Theorem 2 and 3 . Then as in the proof of Theorem 1, we have that $G_{1}^{\Omega}$ is solvable and $G_{1}^{\Omega}$ is 2-transitive on $\Omega-\{C\}$.

Let $K$ be the kernel of the projection $\pi: \operatorname{Aff}(L) \rightarrow\left(\operatorname{GL}(2, \mathbb{Z})\right.$ or $\left.(\mathbb{Z} / 2 \mathbb{Z})^{2}\right)$. Then $K \cap G_{1}$ is an abelian normal subgroup of $G_{1}$ that projects to an abelian normal subgroup $N$ of $G_{1}^{\Omega}$.

Assume first that $N$ is trivial. Then the finite subgroup $G_{1} /\left(K \cap G_{1}\right) \cong G_{1} K / K$ of $\mathrm{GL}(2, \mathbb{Z})$ or $(\mathbb{Z} / 2 \mathbb{Z})^{2}$ projects onto $G_{1}^{\Omega}$. Hence, $G_{1}^{\Omega}$ is isomorphic to a subgroup of a dihedral group of order 8 or 12 by Lemma 2 . Therefore $G_{1}^{\Omega}$ is a cyclic group of order 2,3,4,6 or a dihedral group of order 4,6,8,12. By Lemma 4(5), the group $G_{1}^{\Omega}$ has a unique nontrivial abelian normal subgroup. Hence, $G_{1}^{\Omega}$ is either a group of order 2 or 3 or a dihedral group of order 6 .

Suppose that $G_{1}^{\Omega}$ has order 2. Then $G_{1}^{\Omega}$ has degree 2, and so the degree of $G^{\Omega}$ is 3 by Theorem 9.1 of [14]. Hence $M$ has 3 cusps and $G^{\Omega}=S_{3}$.

Suppose that $G_{1}^{\Omega}$ has order 3. Then $G_{1}^{\Omega}$ has degree 3. Now 6 divides $\left|G_{1}^{\Omega}\right|$ by Theorem 9.3 of [14], which is a contradiction. Hence, this case does not occur.

Suppose that $G_{1}^{\Omega}$ is a dihedral group of order 6 . Then $G_{1}^{\Omega}$ has degree 3, and so $G_{1}^{\Omega}=S_{3}$. Hence, $G_{1}^{\Omega}$ is 3-transitive. Therefore $G^{\Omega}$ is 4 -transitive by Theorem 9.1 of [14], but $G^{\Omega}$ is not 4 -transitive by assumption, and so this case does not occur. 
Now assume that $N$ is nontrivial. Then $N$ is an elementary abelian $p$ group of order $p^{m}=\operatorname{deg}\left(G_{1}^{\Omega}\right)$ for some prime number $p$ and some positive integer $m$ by Lemma 4 . The order of $G_{1}^{\Omega}$ is divisible by $p^{m}\left(p^{m}-1\right)$ by Theorem 9.3 of [14]. The finite group $G_{1} /\left(K \cap G_{1}\right) \cong G_{1} K / K$ is isomorphic to a subgroup of $\operatorname{GL}(2, \mathbb{Z})$ or $(\mathbb{Z} / 2 \mathbb{Z})^{2}$, and $G_{1} /\left(K \cap G_{1}\right)$ projects onto $G_{1}^{\Omega} / N$. Therefore $p^{m}-1 \leq\left|G_{1}^{\Omega} / N\right| \leq 12$. The group $K \cap G_{1}$ has rank at most 2 by Lemma 6 , and $K \cap G_{1}$ projects onto $N$. Therefore $m=\operatorname{rank}(N)$ is 1 or 2 .

Assume first that $m=1$. Then $G_{1}^{\Omega}$ has degree $p$. The solvable 2-transitive finite permutation groups were classified by Huppert [6] (see also $\S 7$ of Chapter XII of [7]). Let $F_{q}$ be the finite field of order $q$. According to Huppert [6] and by Theorem 12 in Chapter VII of [8], the group $G_{1}^{\Omega}$ is isomorphic to a subgroup of the group of affine transformation of the vector space $F_{p}$ over $F_{p}$,

$$
\Gamma(p)=\left\{\alpha: F_{p} \rightarrow F_{p}: \alpha(x)=a x+b \text { with } a, b \in F_{p} \text { and } a \neq 0\right\} .
$$

The group $\Gamma(p)$ is an extension of the additive group $F_{p}^{+}$of the field $F_{p}$ by the multiplicative group $F_{p}^{*}$ of $F_{p}$, that is, we have an short exact sequence

$$
0 \rightarrow F_{p}^{+} \stackrel{\iota}{\longrightarrow} \Gamma(p) \stackrel{\eta}{\longrightarrow} F_{p}^{*} \rightarrow 1 .
$$

where $\iota(b)=x+b$ and $\eta(a x+b)=a$. Therefore $|\Gamma(p)|=p(p-1)$. As $\left|G_{1}^{\Omega}\right| \geq p(p-1)$, we have that $G_{1}^{\Omega} \cong \Gamma(p)$. Hence $G_{1}^{\Omega} / N \cong F_{p}^{*}$, and so $G_{1}^{\Omega} / N$ is a cyclic group of order $p-1$ by Theorem 11 of Chapter VII of [8].

The group $G_{1} /\left(K \cap G_{1}\right)$ is either a finite cyclic group of order at most 6 or a finite dihedral group of order at most 12 . As $G_{1} /\left(K \cap G_{1}\right)$ projects onto $G_{1}^{\Omega} / N$, we have that $G_{1}^{\Omega} / N$ is a cyclic group of order at most 6 . Therefore $p=2,3,5$ or 7 .

Assume that $p=2$, then $G_{1}^{\Omega}$ has order 2. Then $G_{1}^{\Omega}$ has degree 2, and so the degree of $G^{\Omega}$ is 3 by Theorem 9.1 of [14]. Hence $M$ has 3 cusps and $G^{\Omega}=S_{3}$. For an example of such a manifold, see Example 3 in $\S 6$.

Assume that $p=3$. Then $G_{1}^{\Omega}$ has degree 3 and $G_{1}^{\Omega}=S_{3}$. Hence $G_{1}^{\Omega}$ is 3 transitive. Therefore $G^{\Omega}$ is 4 -transitive by Theorem 9.1 of [14]. However $G^{\Omega}$ is not 4 -transitive by assumption. Therefore the case $p=3$ does not occur.

Assume that $p=5$. Then the degree of $G^{\Omega}$ is 6 , and so $M$ has 6 cusps. As $\left|G_{1}^{\Omega}\right|=5 \cdot 4$, we have that $\left|G^{\Omega}\right|=6 \cdot 5 \cdot 4$. Therefore $G^{\Omega}$ is sharply 3 -transitive. By Theorem 2.6 of Chapter XI of [7], we have that $G^{\Omega}=\operatorname{PGL}(2,5)$. For an example of such a manifold, see Example 4 in $\S 6$.

Assume that $p=7$. Then the degree of $G^{\Omega}$ is 8 , and so $M$ has 8 cusps. As $\left|G_{1}^{\Omega}\right|=7 \cdot 6$, we have that $\left|G^{\Omega}\right|=8 \cdot 7 \cdot 6$. Therefore $G^{\Omega}$ is sharply 3 -transitive. By Theorem 2.6 of Chapter XI of [7], we have that $G^{\Omega}=\operatorname{PGL}(2,7)$. For an example of such a manifold, see Example 5 in $\S 6$.

Now assume that $m=2$. As $p^{2}-1 \leq 12$, we have that $p=2$ or 3 .

Assume that $p=2$. Then $G_{1}^{\Omega}$ is a 2 -transitive permutation group of degree $p^{2}=4$ and order a multiple of $p^{2}\left(p^{2}-1\right)=12$. Hence $G_{1}^{\Omega}$ is either $A_{4}$ or $S_{4}$. If $G_{1}^{\Omega}$ were $S_{4}$, then $G_{1}^{\Omega}$ would be 4 -transitive and $G^{\Omega}$ would be 5 -transitive, which is contrary to assumption. Therefore $G_{1}^{\Omega}=A_{4}$. Now $G^{\Omega}$ has degree 5 and has order $5\left|G_{1}^{\Omega}\right|=60$. Hence $M$ has 5 cusps and $G^{\Omega}=A_{5}$. For an example of such a manifold, see Example 6 in $\S 6$.

The remaining part of the proof is devoted to proving that the case $m=2$ and $p=3$ does not occur. On the contrary, assume $m=2$ and $p=3$. Then the order of $G_{1}^{\Omega} / N$ is a multiple of $p^{2}-1=8$. As $\left|G_{1}^{\Omega} / N\right| \leq 12$, we have that $\left|G_{1}^{\Omega} / N\right|=8$. 
Hence $\left|G_{1}^{\Omega}\right|=p^{2}\left(p^{2}-1\right)=72$. From Table 7.3 of [2], we deduce that either $G_{1}^{\Omega}$ is isomorphic to a subgroup of

$$
\Gamma(9)=\left\{\alpha: F_{9} \rightarrow F_{9}: \alpha(x)=a x^{3^{c}}+b \text { with } a, b \in F_{9}, a \neq 0 \text { and } c=0,1\right\} .
$$

or $\left|G_{1}^{\Omega} / N\right| \geq|\mathrm{SL}(2,3)|$. As $|\mathrm{SL}(2,3)|=24>8$, the latter case is not possible. Therefore $G_{1}^{\Omega}$ is isomorphic to a subgroup of $\Gamma(9)$.

Consider the following subgroup of $\Gamma(9)$ :

$$
\Gamma \mathrm{L}(1,9)=\left\{\alpha: F_{9} \rightarrow F_{9}: \alpha(x)=a x^{3^{c}} \text { with } a \in F_{9}^{*} \text { and } c=0,1\right\} .
$$

We have a short exact sequence

$$
0 \rightarrow F_{9}^{+} \stackrel{\iota}{\longrightarrow} \Gamma(9) \stackrel{\eta}{\longrightarrow} \Gamma \mathrm{L}(1,9) \rightarrow 1 .
$$

where $\iota(b)=x+b$ and $\eta\left(a x^{3^{c}}+b\right)=a x^{3^{c}}$. Moreover, we have a short exact sequence

$$
0 \rightarrow F_{9}^{*} \stackrel{\kappa}{\longrightarrow} \Gamma \mathrm{L}(1,9) \stackrel{\rho}{\longrightarrow} \mathbb{Z} / 2 \mathbb{Z} \rightarrow 1
$$

where $\kappa(a)=a x$ and $\rho\left(a x^{3^{c}}\right)=c \mathbb{Z}$, since the Frobenius automorphism $x^{3}$ of $F_{9}$ has order 2. Therefore $\Gamma(9)$ is a solvable group of order $9 \cdot 8 \cdot 2=144$.

The group $\Gamma(9)$ acts effectively on the set $F_{9}$, by evaluation, as a 2 -transitive permutation group. Hence $\Gamma(9)$ has a unique, nontrivial, abelian, normal subgroup of order 9 by Lemma $4(5)$. Let $T=\left\{x+b: b \in F_{9}\right\}$ be the translation subgroup of $\Gamma(9)$. Then $T$ has order 9 and $T$ is the unique, nontrivial, abelian, normal subgroup of $\Gamma(9)$.

Let $\Gamma_{0}(9)$ be the stabilizer of 0 . Then

$$
\Gamma_{0}(9)=\left\{a x^{3^{c}}: a \in F_{9}^{*} \text { and } c=0,1\right\}=\Gamma \mathrm{L}(1,9) .
$$

The group $\Gamma_{0}(9)$ acts transitively on $F_{9}^{*}$ by Theorem 9.1 of [14]. By Theorem 11.2 of [14], the induced action of $\Gamma_{0}(9)$ on $T-\{x\}$ by conjugation corresponds to the action of $\Gamma_{0}(9)$ on $F_{9}^{*}$; indeed, with respect to multiplication given by composition of polynomial functions, we have that

$$
(a x)(x+b)(a x)^{-1}=(a x)(x+b)\left(a^{-1} x\right)=(a x)\left(a^{-1} x+b\right)=x+a b,
$$

and

$$
\left(x^{3}\right)(x+b)\left(x^{3}\right)^{-1}=\left(x^{3}\right)(x+b)\left(x^{3}\right)=\left(x^{3}\right)\left(x^{3}+b\right)=x+b^{3} .
$$

Let $H$ be a subgroup of $\Gamma(9)$ that is isomorphic to $G_{1}^{\Omega}$. Then the order of $H$ is 72 , and so $H$ has index 2 in $\Gamma(9)$. Let $A$ be the unique, nontrivial, abelian, normal subgroup of $H$. Then $A$ has order 9 . We claim that $A=T$. On the contrary, suppose $A \neq T$. Then $A \cap T$ is an abelian normal subgroup of $H$ properly contained in $A$. Therefore $A \cap T=\{x\}$, and so $A$ injects into the group $\Gamma(9) / T$ of order 16 , which is not the case, since $A$ has order 9 . Therefore $A=T$ as claimed.

The group $G_{1}^{\Omega}$ acts transitively on $\Omega-\{C\}$ by Theorem 9.1 of [14]. Hence, the induced action of $G_{1}^{\Omega}$ on $N-\{1\}$ by conjugation is transitive by Theorem 11.2 of [14]. Therefore, the induced action of $H$ on $T-\{x\}$ by conjugation is transitive. Now the induced action of $T$ on $T-\{x\}$ by conjugation is trivial, since $T$ is abelian. The group $H \cap \Gamma_{0}(9)$ is a set of coset representatives for $T$ in $H$, and so the induced action of $H \cap \Gamma_{0}(9)$ on $T-\{x\}$ by conjugation is transitive. As $x^{3}$ acts trivially on $x+1$ by conjugation and $(a x)(x+1)(a x)^{-1}=x+a$, we conclude that

$$
\left\{a \in F_{9}^{*}: a x^{3^{c}} \in H \text { for some } c=0,1\right\}=F_{9}^{*} .
$$

The group $F_{9}^{*}$ is cyclic by Theorem 11 of [8]. 


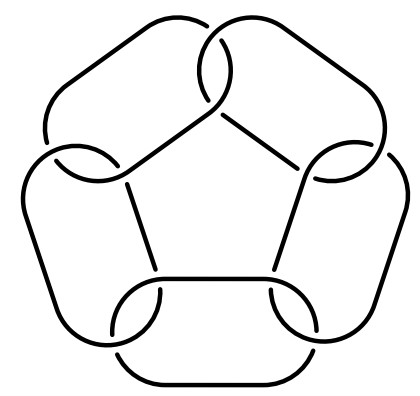

FiguRE 1. A minimally twisted 5-link chain

Assume that $H$ contains $a x$ for some generator $a$ of $F_{9}^{*}$. Then $H \cap \Gamma_{0}(9)$ and $H / T$ are cyclic groups of order 8 . Now assume that $H$ does not contain ax for some generator $a$ of $F_{9}^{*}$. Suppose that $a$ is a generator of $F_{9}^{*}$. Then the generators of $F_{9}^{*}$ are $a, a^{3}, a^{5}, a^{7}$, and so $H$ contains $a x^{3}, a^{3} x^{3}, a^{5} x^{3}, a^{7} x^{3}$. As $\left(a x^{3}\right)^{2}=a^{4} x$, $\left(a x^{3}\right)\left(a^{3} x^{3}\right)=a^{2} x,\left(a x^{3}\right)\left(a^{5} x^{3}\right)=x$ and $\left(a x^{3}\right)\left(a^{7} x^{3}\right)=a^{6} x$, we have that

$$
H \cap \Gamma_{0}(9)=\left\{x, a x^{3}, a^{2} x, a^{3} x^{3}, a^{4} x, a^{5} x^{3}, a^{6} x, a^{7} x^{3}\right\} .
$$

Now $\left\langle a x^{3}\right\rangle=\left\{x, a x^{3}, a^{4} x, a^{5} x^{3}\right\}$ and $\left\langle a^{2} x\right\rangle=\left\{x, a^{2} x, a^{4} x, a^{6} x\right\}$ are distinct cyclic groups of order 4. Therefore $H \cap \Gamma_{0}(9)$ is a quaternion group. Hence $H / T$ and $G_{1}^{\Omega} / N$ are quaternion groups. Thus, in either case $G_{1}^{\Omega} / N$ is not a dihedral group.

Now, the group $G_{1} /\left(K \cap G_{1}\right)$ projects onto $G_{1}^{\Omega} / N$ and $G_{1} /\left(K \cap G_{1}\right)$ is either a cyclic group of order at most 6 or a dihedral group of order $4,6,8$ or 12 . As $G_{1}^{\Omega} / N$ is not a dihedral group, $G_{1}^{\Omega} / N$ must be the image of a dihedral group of order 12 . As 8 does not divide 12, we have a contradiction. Therefore, the case $m=2$ and $p=3$ does not occur.

\section{EXAmples}

In this section, we consider examples of multiply transitive cusped hyperbolic 3-manifolds that satisfy Theorems 2, 3, and 4. All the examples are orientable. Most of our examples are tessellated by isometric, ideal, regular polyhedra so that any face of a polyhedron is the face of exactly two polyhedra of the tessellation. Such a tessellation is called a Platonic tessellation.

A flag of a Platonic tessellation is a triple $(P, F, E)$ consisting of a polyhedron $P$ of the tessellation, a face $F$ of $P$, and an edge $E$ of $F$. We say that an isometry $\phi$ acts on a flag $(P, F, E)$ if $(\phi(P), \phi(F), \phi(E))$ is also a flag.

Example 1. According to Thurston [12], the complement in $S^{3}$ of the minimally twisted 5-link chain shown in Figure 1 is a 5-transitive hyperbolic 3-manifold $M$ of volume $10.149 \ldots$... SnapPy [3] shows that $M$ has a Platonic tessellation consisting of 10 ideal regular tetrahedra. The group $G$ of isometries of $M$ acts transitively on the set of flags of this tessellation so that the stabilizer of each flag has order 2 . Hence $G$ has order 240 . The group $G$ is $(\mathbb{Z} / 2 \mathbb{Z}) \times S_{5}$. The generator of the $\mathbb{Z} / 2 \mathbb{Z}$ factor is orientation-preserving and acts trivially on the set $\Omega$ of cusps of $M$, and the $S_{5}$ factor acts 5-transitively on $\Omega$. The group of orientation-preserving isometries of $M$ acts only 3 -transitively on $\Omega$. 


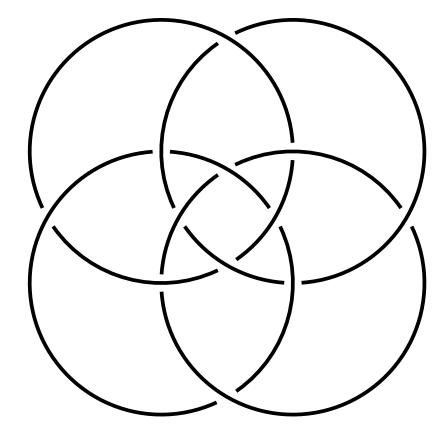

Figure 2. A link whose complement in $S^{3}$ is 4-transitive

Example 2. According to Vogeler [13], the complement in $S^{3}$ of the link shown in Figure 2 is a 4 -transitive hyperbolic 3-manifold $M$. Using SnapPy [3], we found that $M$ has volume $24.092 \ldots$ and the group $G$ of isometries of $M$ is $(\mathbb{Z} / 2 \mathbb{Z}) \times S_{4}$. The $\mathbb{Z} / 2 \mathbb{Z}$ factor of $G$ acts trivially on $\Omega$, and the $S_{4}$ factor of $G$ acts 4 -transitively on $\Omega$.

Example 3. According to Thurston [11], the complement in $S^{3}$ of the Borromean rings shown in Figure 3 is a hyperbolic 3-manifold $M$ of volume 7.327 ... that has a Platonic tessellation consisting of 2 ideal regular octahedra. The group $G$ of isometries of $M$ acts freely and transitively on the set of flags of this tessellation. Hence $G$ has order 48 .

The group $G$ is a semi-direct product $(\mathbb{Z} / 2 \mathbb{Z})^{3} \rtimes S_{3}$ with $S_{3}$ acting 3-transitively on the standard basis of the vector space $(\mathbb{Z} / 2 \mathbb{Z})^{3}$. The subgroup of $G$ that stabilizes both octahedra is the semi-direct product $(\mathbb{Z} / 2 \mathbb{Z})^{3} \rtimes C_{3}$ where $C_{3}$ is the cyclic subgroup of $S_{3}$ of order 3 .

If we consider the ideal regular octahedron $P$ in the conformal ball model $B^{3}$ of hyperbolic 3 -space with ideal vertices $\pm e_{1}, \pm e_{2}, \pm e_{3}$, where $\left\{e_{1}, e_{2}, e_{3}\right\}$ is the standard basis of $\mathbb{R}^{3}$, then the action of the standard basis vectors of $(\mathbb{Z} / 2 \mathbb{Z})^{3}$ on either octahedra of the tessellation of $M$ correspond to the action of the reflections in the coordinate hyperplanes of $B^{3}$ on $P$.

The elementary group $(\mathbb{Z} / 2 \mathbb{Z})^{3}$ acts trivially on the set $\Omega$ of cusps of $M$. The quotient group $G^{\Omega}=S_{3}$ acts 3 -transitively on $\Omega$.

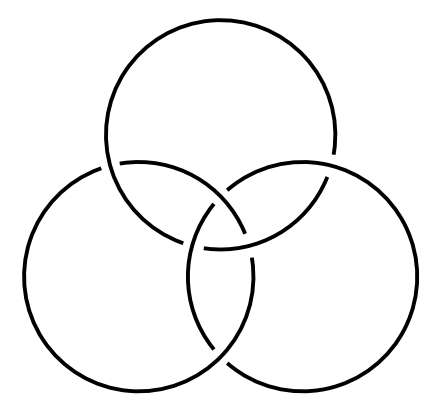

Figure 3. The Borromean rings 


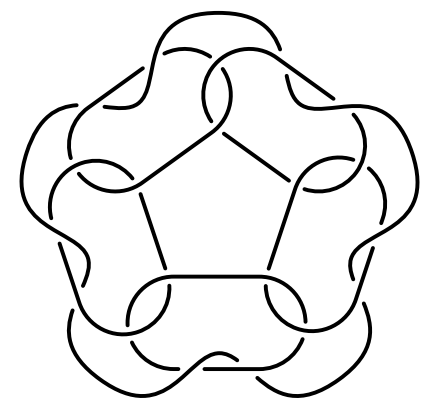

FiguRE 4. The congruence link $(1,\langle 2+i\rangle)$

Example 4. According to Goerner [4], the complement in $S^{3}$ of the link shown in Figure 4 is a hyperbolic 3-manifold $M$ that has a Platonic tessellation consisting of 5 ideal regular octahedra. The volume of $M$ is $18.319 \ldots$ The group $G$ of isometries of $M$ acts freely and transitively on the set of flags of this tessellation. Hence $G$ has order 120. Every isometry of $M$ is orientation-preserving. The group $G$ acts effectively and sharply 3-transitively on the set of 6 cusps of $M$, and therefore $G=\operatorname{PGL}(2,5)$ by Theorem 2.6 of Chapter XI of [7]. Although $G \cong S_{5}$, we write $G=\operatorname{PGL}(2,5)$ to indicate that $G$ is a degree 6 permutation group according to its natural representation as a permutation group on the set of the six 1-dimensional vector subspaces of the vector space $F_{5} \oplus F_{5}$ over the field $F_{5}$.

Example 5. According to Thurston [12], the complement in $S^{3}$ of the link shown in Figure 5 is a hyperbolic 3-manifold $M$ with extraordinary 336-fold symmetry and volume $28.418 \ldots$. According to Goener [4], the manifold $M$ has a Platonic tessellation consisting of 28 ideal regular tetrahedra, and the group $G$ of isometries of $M$ acts freely and transitively on the set of flags of this tessellation. Hence $G$ has order 336. Every isometry of $M$ is orientation-preserving. The group $G$ acts effectively and sharply 3-transitively on the set of 8 cusps of $M$, and so $G=$ $\operatorname{PGL}(2,7)$ by Theorem 2.6 of Chapter XI of [7].

Example 6. Our last example is an orientable hyperbolic 3-manifold $M$ that is obtained by gluing together two ideal regular dodecahedra along their faces. Each face of the first dodecahedron is glued to the opposite face of the second

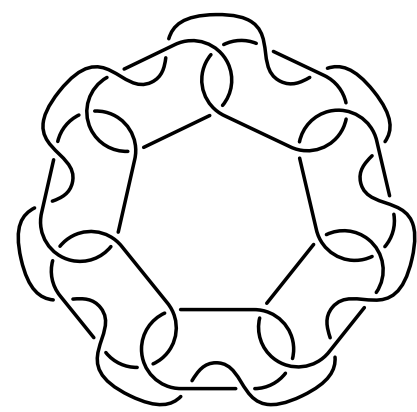

FiguRE 5. Thurston's congruence link 
dodecahedron with a twist of $\pi / 5$ as in Figure 10.1.1 of [9]. The manifold $M$ has volume $41.160 \ldots$ and exactly 5 cusps. The group $G$ of isometries of $M$ acts freely and transitively on the set of flags of this Platonic tessellation. Hence $G$ has order 120. Every isometry of $M$ is orientation-preserving. This implies that $M$ is manifold odode02_00912 in Goerner's census of double-dodecahedral cusped hyperbolic 3-manifolds (see Tables 4 and 10 of [5]). The manifold $M$ is a homology link, but $M$ is not the complement of a link in $S^{3}$ by the main theorem of Goener's paper [4].

The group $G$ is $(\mathbb{Z} / 2 \mathbb{Z}) \times A_{5}$. The subgroup of $G$ that stabilizes both dodecahedra is the $A_{5}$ factor of $G$. The generator of the $\mathbb{Z} / 2 \mathbb{Z}$ factor of $G$ acts trivially on the set $\Omega$ of cusps of $M$, and the $A_{5}$ factor of $G$ acts effectively and sharply 3-transitively on $\Omega$.

\section{Final Remarks}

We conclude with some natural problems that this paper suggests.

Problem 1: Classify all the hyperbolic 3-manifolds that satisfy Theorems 2, 3, or 4 .

In this regard, we ask the following more specific questions:

Question 1: Is the complement in $S^{3}$ of the minimally twisted 5-link chain the only hyperbolic 3 -manifold that satisfies Theorem 2 ?

Question 2: Are there any nonorientable hyperbolic 3-manifolds that satisfy Theorems 2,3 , or 4 ?

Problem 2: Determine the possible numbers of cusps of 2-transitive cusped hyperbolic 3-manifolds of finite volume.

According to Vogeler [13], there exists a 2-transitive hyperbolic 3-manifold of finite volume with exactly $q$ cusps for each prime power $q$.

\section{Acknowledgments}

We thank Matthias Goerner for providing us with graphic files for Figures 1, 4, and 5, that first appeared in [1], and for helpful communications concerning his hyperbolic 3-manifold odode02_00912.

\section{REFERENCES}

[1] M. D. Baker, M. Goerner, and A. W. Reid, All known principal congruence links, arXiv:1902.04426v1 [math.GT] 11 Feb 2019.

[2] P. J. Cameron, Permutation Groups, London Math. Soc. Student Texts 45, Cambridge Univ. Press, Cambridge, UK, 1999.

[3] M. Culler, N. M. Dunfield, M. Goerner, and J. R. Weeks, SnapPy, a computer program for studying the geometry and topology of 3-manifolds, http://snappy.computop.org.

[4] M. Görner, Regular tessellation link complements, Exp. Math. 24 (2015), 225-246.

[5] M. Goener, A census of hyperbolic Platonic manifolds and augmented knotted trivalent graphs, New York J. Math. 23 (2017), 527-553.

[6] B. Huppert, Zweifach transitive, auflösbare Permutationsgruppen, Math. Z. 68 (1957), 126150. 
[7] B. Huppert and N. Blackburn, Finite Groups III, Springer-Verlag, Berlin, Heidelberg, and New York, 1982.

[8] S. Lang, Algebra, Addison-Wesley, Reading, Mass., 1965.

[9] J. G. Ratcliffe, Foundations of Hyperbolic Manifolds, Second Edition, Graduate Texts in Math., vol. 149, Springer-Verlag, Berlin, Heidelberg, and New York, 2006.

[10] J. G. Ratcliffe and S. T. Tschantz, On the isometry group of a compact flat orbifold, Geom. Dedicata 177 (2015), 43-60.

[11] W. P. Thurston, The Geometry and Topology of Three-Manifolds, Lecture Notes, Princeton University, Princeton, NJ, 1980.

[12] W. P. Thurston, How to see 3-manifolds, Class. Quantum Grav. 15 (1998), 2545-2571.

[13] R. Vogeler, Cusp transitivity in hyperbolic 3-manifolds, arXiv:1906.07147v1 [math.GT] 17 Jun 2019.

[14] H. Wielandt, Finite Permutation Groups, Academic Press, New York, 1964.

Department of Mathematics, Vanderbilt University, Nashyille, TN 37240

E-mail address: j.g.ratcliffe@vanderbilt.edu 